\title{
RESEARCH ON THE CONTROL POINT EXTRACTION METHOD OF CHINESE GAOFEN 7 SATELLITE USING ALTIMETER AND FOOTPRINT CAMERA
}

\author{
Binbin $\mathrm{Li}^{1}$, Huan Xie ${ }^{1,}$, Xiaohua Tong ${ }^{1}$, Yinqiao Cai ${ }^{2}$, Zhijie Zhang ${ }^{1,3}$ \\ ${ }^{1}$ College of Surveying and Geo-informatics, Tongji University, Shanghai 200082, China \\ ${ }^{2}$ Shanghai Institute of Technical Physics, Chinese Academy of Sciences, Shanghai 200083, China \\ ${ }^{3}$ School of Surveying and Land Information Engineering, Henan Polytechnic University, Jiaozuo 454000, \\ China \\ *Email:huanxie@tongji.edu.cn
}

\begin{abstract}
Satellite laser altimetry is one of the most advanced information acquisition technologies in Earth observation system. It can provide highaccuracy elevation information, however, due to the lack of detail intensity information, its planimetric accuracy is usually worse than the elevation accuracy. Gaofen 7 (GF-7) satellite scheduled for launch in 2019 will be equipped with laser altimeter, footprint camera, stereo mapping camera, etc. The laser altimeter together with the footprint camera was designed to provide high accuracy ground control point of satellite mapping. The laser altimeter can provide the highaccuracy elevation information and the joint processing of footprint camera and stereo mapping camera can provide high-accuracy planimetric information. Therefore, this paper mainly studies the technology of extracting highaccuracy control points based on GF-7 satellite's altimeter, footprint camera and stereo mapping camera using a simulated dataset extracted from Quickbird image and ICESat altimetric data.
\end{abstract}

\section{INTRODUCTION}

Satellite laser altimetry has been used in deep space exploration since 1960s because of its highaccuracy range detection capability. In the field of earth science, besides some experimental loads, the laser altimeter GLAS of ICESat satellite ${ }^{[1]}$ launched by NASA in 2003 is the first satelliteborne laser altimeter system in the world and was retired in 2010. As its second generation satellite, ICESat-2 satellite ${ }^{[2]}$ was successfully launched in 2018 and is currently in the testing and calibrating stage.

In addition to the experimental laser loads carried by Ziyuan 3 satellite (ZY-3) launched by China in
May 2016, there is a plan to launch the Gaofen 7 (GF-7) satellite in 2019. GF-7 is a high resolution stereo mapping satellite and its main payloads include laser altimeter, footprint camera and stereo mapping camera. Among them, stereo mapping camera obtains the stereo image of the earth surface under flight conditions and the laser altimeter is used to provide the control point in the stereo image with the assistance of the footprint image imaged by footprint camera. The terrain data onto stereo mapping camera will be corrected by these control points, which can improve the elevation accuracy of the stereo image so as to satisfy the requirement of 1:10000 stereographic mapping under the condition of few control points. Therefore, this paper focuses on the research of the technology of extracting high accuracy control points based on GF-7 load characteristics using a simulated dataset by Quickbird image and ICESat altimetric data.

\section{METHODOLOGY}

The elevation information of control points can be obtained from laser altimetric data, and the planimetric information of control points can be obtained by processing the footprint image and the stereo image.

\subsection{Elevation extraction using laser altimetric data}

At first, the initial distance between the laser altimeter and the laser footprint can be obtained by multiplying the speed of light in a vacuum and half of the time difference between the centroids of the transmitted pulse and the received pulse ${ }^{[3]}$.

And then, the initial laser footprint location can be calculated by using the satellite laser footprint location model ${ }^{[4][5]}$ combined with the initial distance, the satellite position, attitude, the 
rotation matrix from the International Celestial Reference Frame (ICRF) coordinate system to the International Terrestrial Reference Frame (ITRF) coordinate system, the rotation matrix from satellite body coordinate system to ICRF coordinate system and so on.

In order to eliminate the spacecraft velocity influence on laser pointing, relevant resolution strategy ${ }^{[6]}$ that the satellite attitude data at the time of laser launching and the satellite position data at the time of laser reaching the ground are used to participate in the calculation of the laser footprint positioning model has been adopted.

Finally, related geophysical corrections including atmospheric delay correction ${ }^{[7]}$ and tidal correction $^{[8]}$ are made to correct the initial laser footprint location. Through the above process, the elevation accuracy is generally about $14 \mathrm{~cm}$ for ICESat $^{[9][10]}$.

\subsection{Planimetric position extraction by combining footprint image and stereo image}

A large footprint will be formed on the ground when satellite laser altimeter emits laser pulse to the ground with an altitude of approximately 600 $\mathrm{km}$. For example, the diameter of laser footprint produced by ICEsat is approximately $70 \mathrm{~m}$. Since laser altimeter cannot produce the continuous ground intensity information, its planimetric accuracy is usually worse than the elevation accuracy. Different from previous laser altimetric satellite, GF-7 will be equipped with footprint camera. It means that laser footprint planimetric information can obtained by matching footprint image with stereo image, which is a new model of extracting the planimetric information of laser footprint.

Footprint camera images the ground at the time of laser emission and ideally, the center of mass of the footprint in footprint image is the position of the laser footprint. Base on traditional gray weighted centroid algorithm, the centroid of the laser footprint recorded by the footprint camera can be extracted. And then, the stereo image will be re-sampled to the same resolution as the footprint image in order to extract and match feature points between two images. The centroid of the laser footprint in two images can be matched according to their matched relation, as shown in Figure 1. Finally, the planimetric information of control point can be extracted from the position of the centroid of the laser footprint in stereo image.

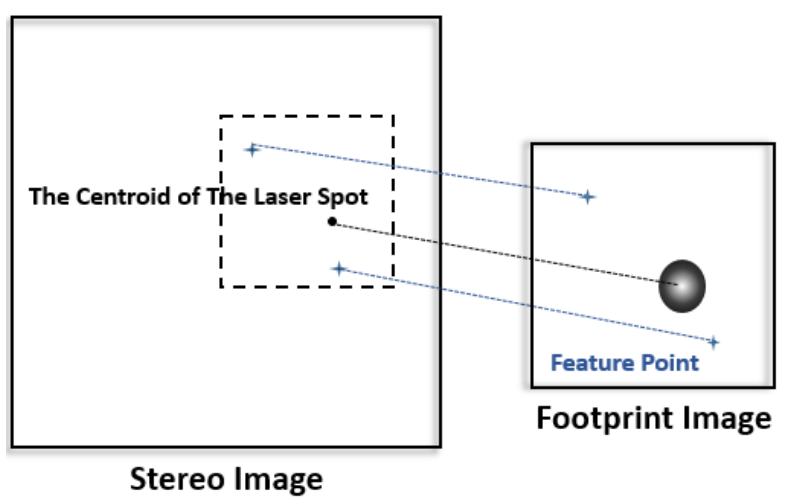

Fig. 1 The matching process of footprint image and stereo image

At present, the accuracy of image registration can reach sub-pixels level, which means that the planimetric information of laser footprint can be obtained at meter level in meter-scale stereo image. Because GF-7 has not yet been launched, this paper used Quickbird image as simulation data to generate stereo image and footprint image. By processing 30 groups of simulation images, image registration accuracy can reach sub-pixel level and the laser footprint planimetric accuracy can reach meter-level.

\section{SUMMARY}

Different from other traditional satellite laser altimeters which are used to observe the earth, GF-7 altimeter will further improve the accuracy of control points for satellite optical stereo image adjustment and stereo mapping because of the assistance of footprint camera. To achieve the goal, this paper researched the process of control point extraction technology for GF-7 with the assistant of footprint image, which will be of some reference value to GF-7 stereo mapping with high accuracy.

\section{ACKNOWLEDGEMENTS}

This work was supported by the National Natural Science Foundation of China under Grant No. 41822106 and 41571407, National High Resolution Ground Observation System of China (GF-7) under Grant No. 11-Y20A12-9001-17/18, 
Science and Technology Innovation Action Plan Program of Shanghai under Grant No. 18511102100, Dawn Scholar of Shanghai under Grant No. 18SG22, and the Fundamental Research Funds for the Central Universities of China.

\section{REFERENCES}

[1] B. E. Schutz, et al. Overview of the ICESat Mission. Geophysical Research Letters, 32(21):L21S01. (2005)

[2] W. Abdalati, et al. The ICESat-2 Laser Altimetry Mission, Proceedings of IEEE, 98(5), 735-751. (2010)

[3] A. C. Brenner, et al. Derivation of Range and Range Distributions from Laser Pulse Waveform Analysis for Surface Elevations, Roughness, Slope, and Vegetation Height. GLAS Algorithm Theoretical Basis Document, Version 3.0. (2011)

[4] B. E. Schutz. Laser Footprint Location (Geolocation) and Surface Profiles. GLAS Algorithm Theoretical Basis Document, Version 3.0. (2002)

[5] X. M. Tang, et al. The rigorous geometric model of satellite laser altimeter and preliminarily accuracy validation. Acta Geodaetica Et Cartographica Sinica. 45(10):1182-1191.(2016)

[6] S. Bae, et al. Precision Attitude Determination (PAD). Geoscience Laser Altimeter System (GLAS) Algorithm Theoretical Basis Document, Version 2.2. [S. 1.]: NASA. (2002)

[7] J. C. Owens, Optical refractive index of air: Dependence on pressure, temperature, and composition, Appl. Opt., 6, 51-59. (1967)

[8] G. Petit, et al. IERS conventions (2010). IERS Technical Note No.36. (2010)

[9] C. A. Shuman, et al. ICESat Antarctic elevation data: Preliminary precision and accuracy assessment. Geophysical Research Letters, 33(7):L07501. (2006)

[10] X. Wang, et al. Earth science applications of ICESat/GLAS: a review. International Journal of Remote Sensing, 32(23):28. (2011) 